\title{
Solute-Induced Perturbations of Solvent-Shell Molecules Observed using Multivariate Raman Curve Resolution
}

\author{
Pradeep Perera, Melanie Wyche, Yvette Loethen and Dor Ben-Amotz*
}

Purdue University, Department of Chemistry, West Lafayette, IN 47907.

\section{Supporting Information}

\section{Experimental Procedure}

Solutions of acetonitrile, acetone, THF or pyridine in water, or acetonitrile or cyclohexane in dichloroethane were prepared from the corresponding pure liquids, either used as received or distilled to reduce interference from fluorescent impurities. All the above solutes/solvent mixtures used in this study are miscible at all concentrations. The solutions are contained in $4 \mathrm{ml}$ round glass vials and the spectra are collected using a home-built back-scattering micro-Raman system, with an air-cooled Ar-ion laser (MellesGriot $514.532 \mathrm{~nm}, 85 \mathrm{~mW}$ at the sample), fiber-coupled to an imaging spectrograph (Acton SpectraPro 300i) with a $600 \mathrm{gr} / \mathrm{mm}$ grating and a liquid $\mathrm{N}_{2}$-cooled CCD detector (Princeton Instruments, $1024 \mathrm{X} 256$ pixel). ${ }^{1}$ A long working distance objective (Olympus, 20X) is focused within the liquid (about $1 \mathrm{~mm}$ past the glass vial wall, so as to assure that the spectra derive only from the solution). Each spectrum is integrated for 100400 seconds and two spectra are combined to strip cosmic spikes and signal average. ${ }^{2}$

\section{Multivariate Curve Resolution Calculation Procedure}

The multivariate curve resolution ${ }^{3}$ algorithm which is used to extract pure solvent and solute-correlated spectra from our experimental solution Raman spectra is implemented as follows (using MATLAB 7.0). The input spectra are pre-processed (to reduce noise) using principal component analysis (PCA), by reconstructing the input spectra from the first two PCA eigenvectors. A non-linear least squares algorithm is used to solve the matrix equation, $\mathbf{D}=\mathbf{C S}^{\mathbf{T}}+\mathbf{E}$, where $\mathbf{D}$ is a matrix composed of input solution spectra (each normalized to unit area), $\mathbf{C}$ contains the output concentrations of the two components, $\mathbf{S}$ contains the output spectra of each component and $\mathbf{E}$ is the residual error (noise). ${ }^{3}$ Both $\mathbf{C}$ and $\mathbf{S}$ are constrained to be non-negative (and to match the pure solvent spectrum at vanishing solute concentration).

\section{Supplementary Figures}

Figure 1S contains raw Raman spectra of a series of aqueous solutions of acetonitrile. The spectra are each normalized to unit area, which is also the pre-processing step required in implementing the multivariate curve resolution algorithm. Before normalization each spectrum had an intensity of approximately 7,000 counts per second at $3400 \mathrm{~cm}^{-1}$ (the pure liquid spectra shown in Fig. 1 each represent the average of 400 spectra).

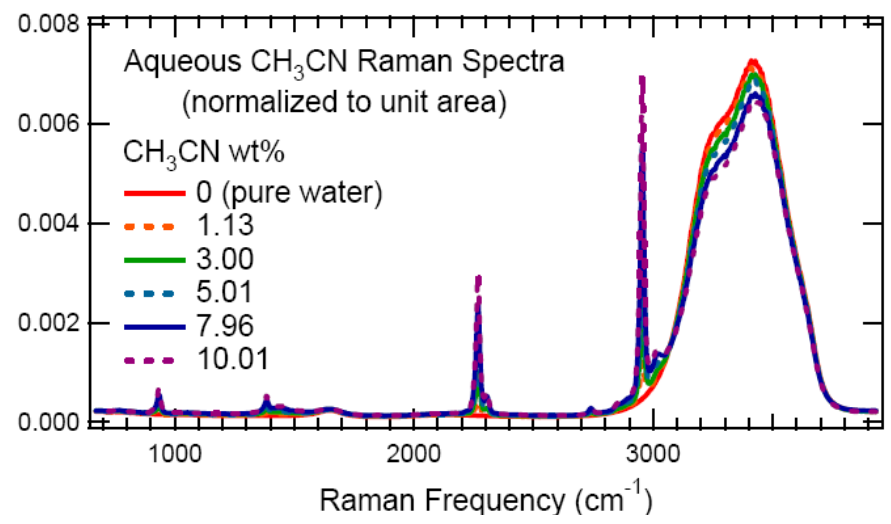

Fig. 1S. Raw Raman spectra of aqueous acetonitrile solutions. 
Figure 2S shows the curve resolved spectra obtained from the raw data shown in Fig. 1S. Each of the resolved curves are again normalized to unit area (but the red curve is scaled down by a factor of 5 for better visibility). These curves are the same as the corresponding curves shown in the upper panels of Fig. 1 in the paper. Note the shifted $\mathrm{OH}$ band that appears in the acetonitrile correlated spectrum, which represents about one water molecule whose spectrum is significantly perturbed by acetonitrile.

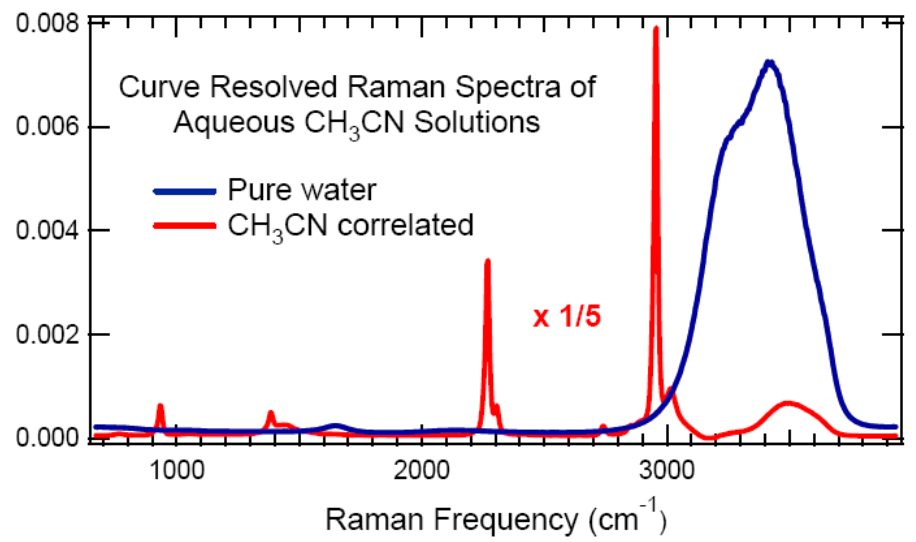

Fig. 2S. Curve resolved spectra obtained from aqueous solutions of acetonitrile.

Figure 3S shows curve resolved spectra obtained from aqueous acetone solutions, which are clearly similar to those obtained for aqueous acetonitrile. Notice in particular the appearance of a perturbed water $\mathrm{OH}$ band associated with the acetone spectrum, again indicating that about one water molecule is shifted to higher frequency by its association with acetone.

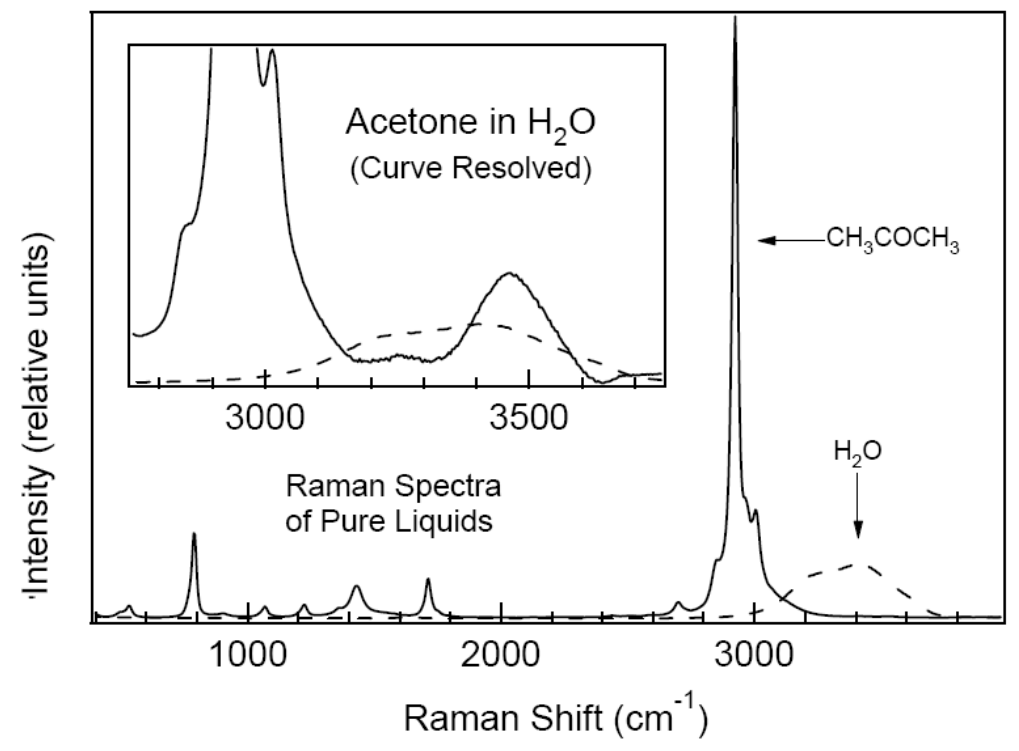

Fig. 3S. Curve resolved Raman spectra obtained from aqueous acetone solution (see Fig. 1 of the paper for details).

Figures 4S and 5S show Raman curve resolved spectra obtained from aqueous solutions of pyridine and tetrahydrofuran (THF), respectively. In each case the solid curves are the solute correlated spectra, the dotted curves are spectra of the pure solute liquid and the dashed curves show the spectrum of pure water. Thus, the approximately equal integrated intensities of the new $\mathrm{OH}$ bands and the pure water $\mathrm{OH}$ band (which is scaled to be approximately equimolar with the solute), indicates that about one water molecule is significantly perturbed by each of these solutes. The results in Figs. 4S and 5S clearly reveal the appearance of two new 
$\mathrm{OH}$ bands in both the pyridine and THF solutions, in contrast to the single new $\mathrm{OH}$ band in the aqueous acetonitrile and acetone solutions (as discussed in the paper).

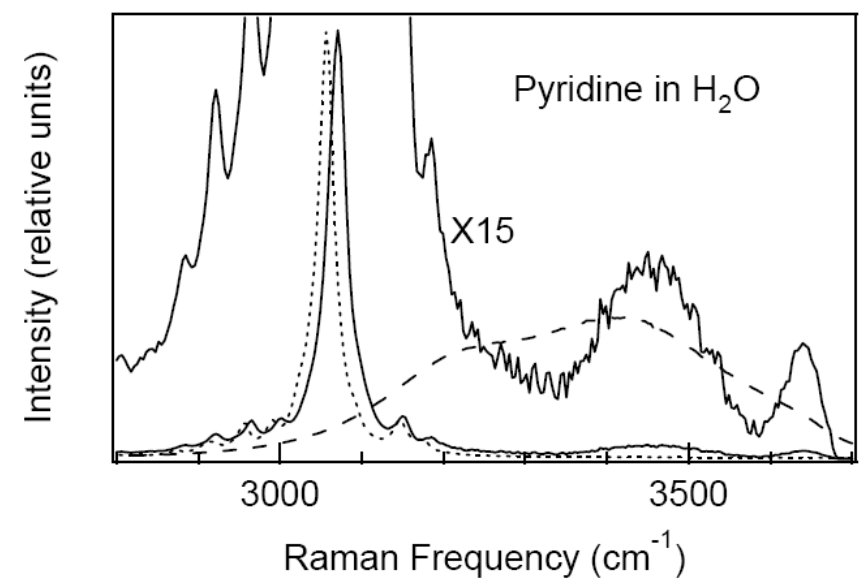

Fig. 4S. Curve resolved spectra of aqueous pyridine solutions (see text for details).

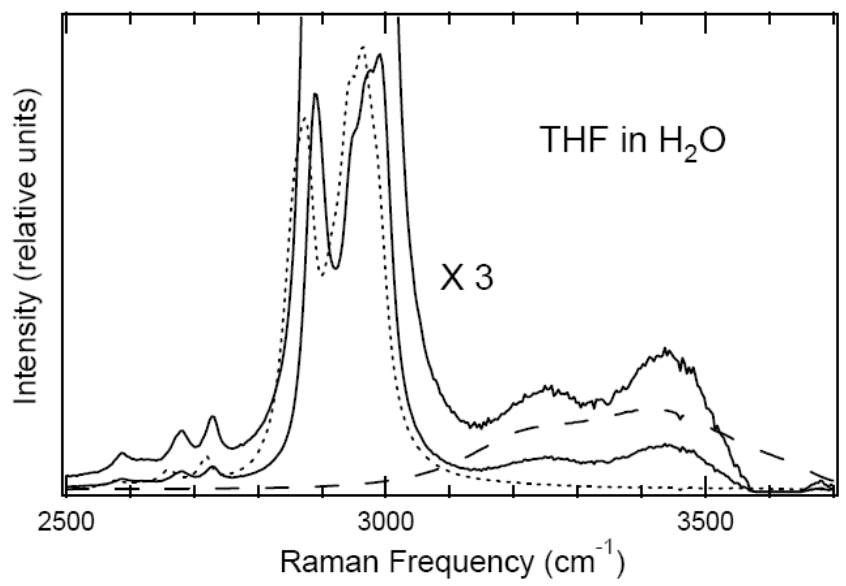

Fig. 5S. Curve resolved spectra of aqueous THF solutions (see text for details).

\section{References}

(1) Xie, Y.; Jiang, Y.; Ben-Amotz, D. Analytical Biochemistry 2005, 343, 223-230.

(2) Zhang, D. M.; Jallad, K. N.; Ben-Amotz, D. Appl Spectrosc 2001, 55, 1523-1531.

(3) Tauler, R. Chemometrics and Intell. Lab. Syst. 1995, 30, 133-146. 